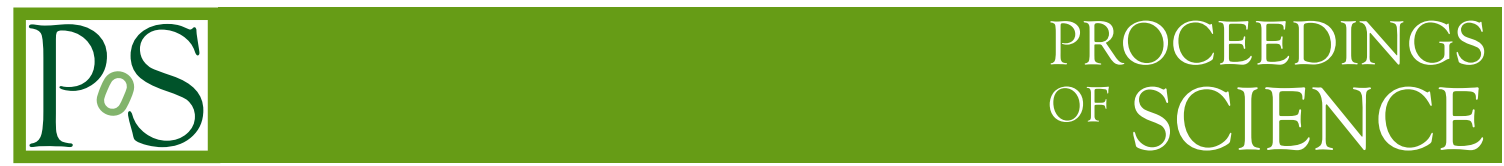

\title{
Report from Young Scientist Fora at LHC
}

\author{
Tamas Almos Vami* and Young Scientist Fora at LHC \\ Johns Hopkins University, \\ Baltimore, USA \\ E-mail: tamas.almos.vami@cern.ch
}

Half of each collaboration at LHC is composed of early career scientists (BSc, MSc, PhD students and PostDocs). To represent this demographic, young scientist committees were created at all LHC experiments. Although the structure and approach in these committees are different, the goals are common: represent the interests of young scientists and provide a forum to gather and discuss topics relevant to the young LHC community. In order to achieve this, several programs are organized either by individual committees or altogether as the young scientist fora. This paper introduces the early career scientist committees, details the programs organized by them and by the young scientist fora, and mentions further plans as well.

The Eighth Annual Conference on Large Hadron Collider Physics-LHCP2020

25-30 May, 2020

online

${ }^{*}$ Speaker 


\section{Introduction}

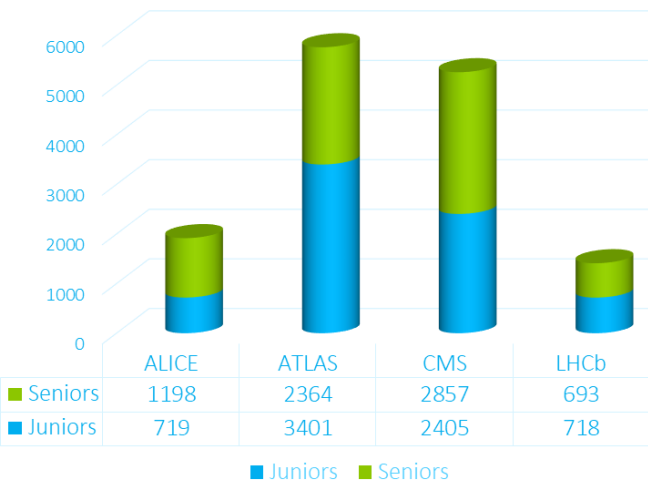

(a)

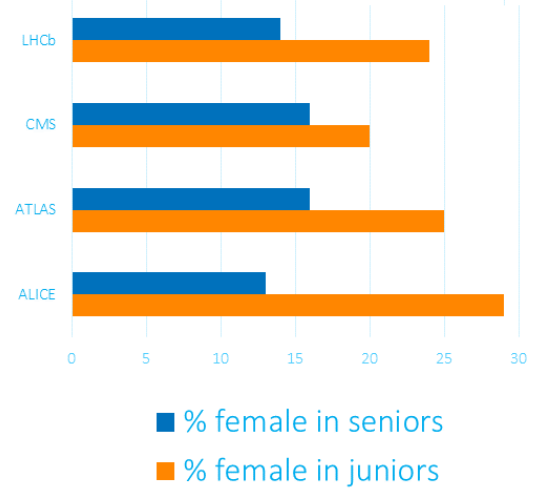

(b)

Figure 1: Demographics at LHC. (a) shows the age categorization, blue represents the number of juniors (BSc, MSc, PhD students and PostDocs), while in green the number of seniors are shown for all the four experiments at LHC. (b) shows the percentage of females divided again into the same two categories.

Figure 1 shows two demographic figures for the four LHC experiments [1-4]. In the 1a plot, each column represents a collaboration. It shows the number of "juniors" (i.e. BSc, MSc, PhD students and PostDocs) in blue, and the number of "seniors" in green. From this plot we can conclude that half of each collaboration is composed of early career scientists.

In the $1 \mathrm{~b}$ plot on the other hand, each row represents a collaboration. In this plot, the percentage of females is shown, divided again into the same two categories. Orange represents the juniors, and dark blue represents the seniors category. We can observe an average about $10 \%$ increase in the number of females in junior category.

To represent this demographic, young scientist committees were created at all LHC experiments. History of the committees starts with the Junior Community in ALICE, which was formed in 2010. The second oldest committee is the CMS Young Scientist Committee (YSC) from 2013. In 2014, LHCb's Early Career, Gender and Diversity Office (ECGD) is created. In the ATLAS Collaboration the Early Career Scientist Board (ECSB) was proposed in 2017.

The structure of these committees is somewhat different. In ALICE there are three Junior Representatives with a mandate for two years. In ATLAS there are seven members with a two year mandate each, with a monthly rotating chair, and half of members change each year. In CMS there are twenty members for one year which can be extended for another year and there is a chair, a deputy and a secretary as coordinators of the team. In LHCb there are two members, usually one female and one male, for two years.

Aim of the young scientist committees are to

a) represent the interest of young scientists,

b) ensure communication between young scientists and management,

c) improve the recognition of work among young scientists, and 
d) provide a forum to gather and discuss topics relevant to the young community

a-c) is achieved somewhat differently in each committee. In ALICE, the Junior Representatives vote in Collaboration + Management Boards $(\mathrm{CB}+\mathrm{MB})$. In ATLAS, ECSB members meet several times a year with management, and the board also sends out regular surveys to the young scientists in order to assess collaboration attitudes. In CMS, the YSC is an "ex officio" member of the Collaboration Board (which means that the chair can attend the Collaboration Board meeting but has no voting rights) and there are quarterly meetings with the spokesperson team and the CB chair team. LHCb's ECGD Office is also a non-voting member of the Collaboration Board; its chairs frequently meet with management and send out surveys to LHCb's early career scientist community.

d) is ensured by several programs organized either by individual committees or altogether by the young scientist fora.

\section{Programs by the individual committees}

All the four committees participate in some kind of Induction Courses for newcomers. These courses are envisaged for newcomers including all levels: from students, to PostDocs, to more experienced collaborators. The goal of these events is to inform the participants about the challenges and fascination of the activities in the collaborations. The talks and discussions are usually presented by the management, the subsystem managers and the coordinators (Figure 2a).

In ATLAS and CMS, there is an event where early career scientists get the opportunity to informally meet and speak with the management and coordinators, called "Meet\&Eat" (Figure 2b) and "Lunch with the professors", respectively.

There are programs meant only for juniors, like ALICE's "Journal Club" or CMS' "Dinner\&Drinks" (Figure 2c), which aim to create an environment for the early career scientists where they can freely express their views, complaints and suggestions, or just to meet their fellow young scientists.

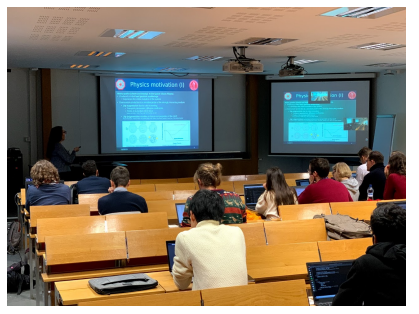

(a)

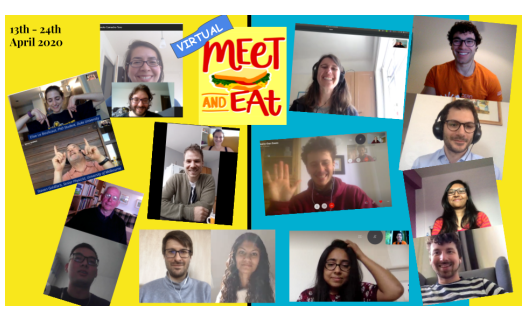

(b)

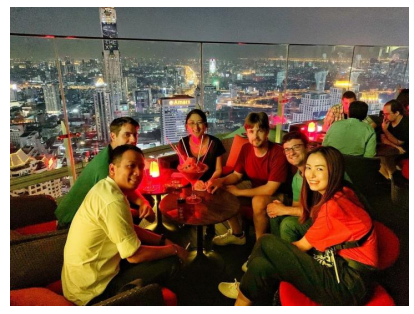

(c)

Figure 2: Photos from programs organized by the individual committees. (a) Introductory lectures in ALICE, (b) Meet\&Eat in ATLAS and (c) Dinner\&Drinks in CMS.

In ALICE there is a "Juniors Diversity Workshop" (Figure 3a) which is an interactive workshop on diversity management concepts and diversity at CERN for juniors only.

The ALICE, ATLAS and LHCb collaborations, with the support of the young scientist committees, organize events for improving analysis skills. In ATLAS there are "Analysis tutorials" while ALICE and LHCb have a shared "Analysis Starterkit" event (Figure 3b). 
All collaborations offer the possibility to show the work of young scientists in form of talks or posters, usually during collaboration weeks. In ALICE, there is a "Junior's Day" organized every ALICE week. At this event juniors can show their scientific presentations, followed by an invited educative talk by a senior expert and a dinner for the Juniors. During the off-site CMS week, there is poster competition for the young scientists (Figure 3c). The winner of this competition can present their work at the Weekly General Meeting which is the event meant to inform the whole collaboration. In LHCb, there is a 2 hour session at the LHCb weeks allocated to lectures meant for juniors.

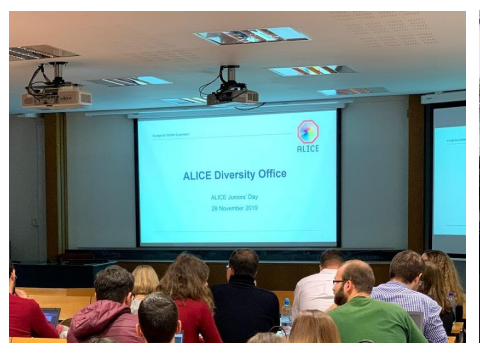

(a)

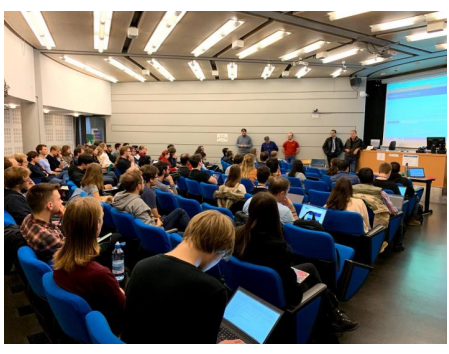

(b)

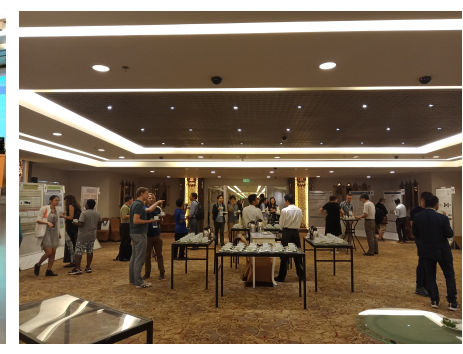

(c)

Figure 3: Photos from programs organized by the individual committees. (a) Juniors Diversity Workshop in ALICE, (b) Analysis Starterkit in ALICE and LHCb and (c) poster session at the CMS Week in Bangkok.

Last year in ATLAS, the ECSB organized a workshop on grant writing (Figure 4a), to improve the soft skills of the young scientists.

CMS' YSC organized its first "Job Matching Event for academia" this year (Figure 4b), in order to help both the young scientists of the CMS Collaboration who wish to pursue a scientific career and apply for a job at another CMS institute, and the senior scientists of the CMS Collaboration who are actively seeking specialized personnel for their groups.

The CMS YSC participated in Reflection Group to revise spokesperson election in 2019 (Figure 4c). In 2020 there was an election, in which $85 \%$ of the eligible scientist have voted, which can be considered as a success for young scientist.

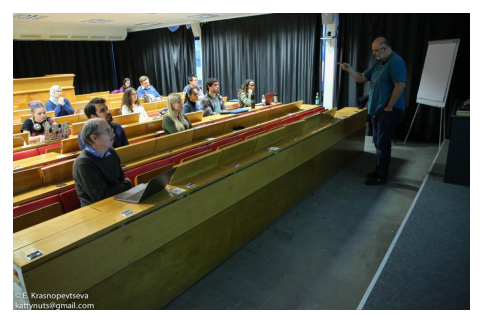

(a)

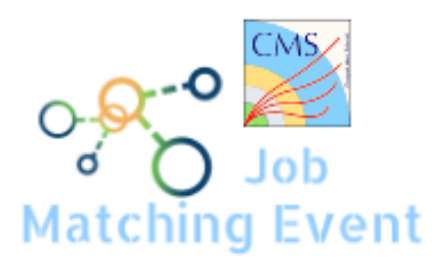

(b)

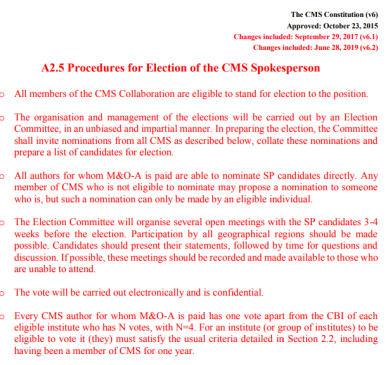

(c)

Figure 4: Photos from programs organized by the individual committees. (a) Workshop on grant writing in ATLAS, (b) Job Matching Event for academia in CMS and (c) CMS constitution showing the changes that allow PostDocs to vote for the Spokesperson. 


\section{Programs by the Young Scientist Fora}

There are several Young Scientist Fora programs that are organized either altogether or rotating yearly between the four experiments. The young scientist committees meet annually (twice a year in the future) to discuss common issues, share tips and best practices, and plan the common programs. This year, we have decided to extend the portfolio of the common programs with a series of soft skill workshops.

The first of these "LHC Soft Skills Workshops" was held on the 15th of April, 2020 [5]. The format of the event is as follows. In the beginning, there are presentations by experts, which is followed by a round table discussion, and then a Q\&A session with all participants. The topic of this first event was about how to make the most of working from home, which was very relevant during COVID-19 times. Although the primary audience was the early career scientist, we opened up the event to any age group. Figure 5 shows two diagrams from the first Soft Skills Workshop in 2020. 5a shows the career level distribution of the registered participants. $46 \%$ of the participants were PhD students, followed by PostDocs with 30\%, and about the same number of seniors and undergraduate students. $5 \mathrm{~b}$ shows the experiments of the participants. Note that besides the four LHC experiment, there were 37 participants from non-LHC experiments.

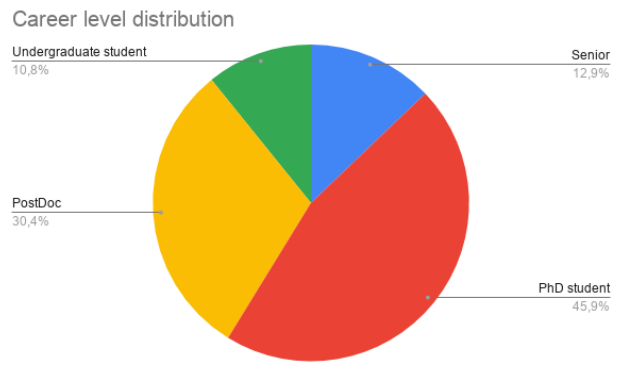

(a)

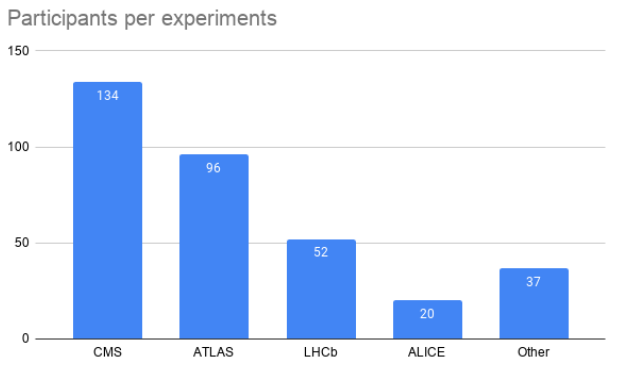

(b)

Figure 5: Diagrams from the first Soft Skills Workshop in 2020. (a) shows the career level distribution of the registered participants, and (b) shows their experiments.

The second event will be held on the 3rd of June, 2020 [6]. It will focus on CV and motivation letter writing for both academia and industry. The number of registered participants is more than 450 .

The idea is to have a soft skill related workshop every three months. Regarding the plans for the further future, CERN Alumni Relations will organize the next version of the event called "Moving out of academia to ... MedTech" [7] in September. This event provides the opportunity to learn more on how CERN alumni successfully managed a transition from Academia to the world of medical technologies. The first part of the event is focused on the nature of the work carried out by the panelists and on other skills they acquired at CERN which have helped them in the transition. The second part of the event will provide the audience with practical advice on how to prepare for such a transition, how to get started, what errors to avoid, where to look for help and how to promote your assets. 
In November, the fora together with the Alumni will organize the next version of the "LHC Career Networking" event. This event offers an insight into career opportunities outside of academia. Various former members of the LHC collaborations give presentations and elaborate on their experience in companies in a diverse range of fields (industry, finance, IT, etc.). At the end of the event, there is a panel discussion which gives the opportunity to ask questions as well (Figure 6) [8]. Next event is planned to be on the 16th of November [9]
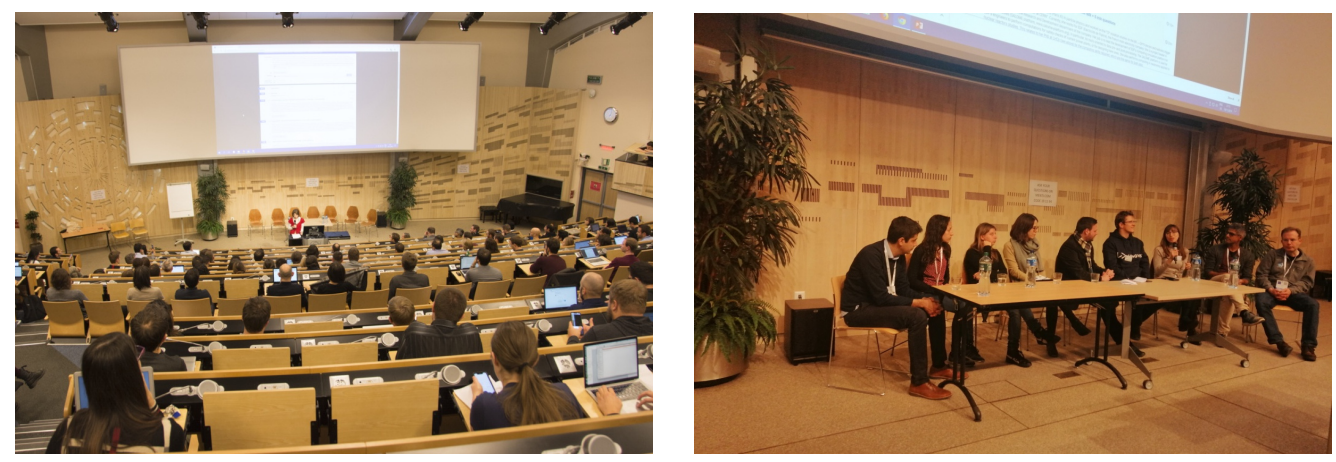

Figure 6: Photos from the networking event in 2019.

One of the programs that the committees organize in a rotating fashion is LHC's "a conversation over ice cream" (Figure 7). For this event the idea is that there are short (15-20 min) talks by experts for a general audience with ice cream (this year with screens only). The first common event was held on the 16th of July, 2018 with the title "A fresh look at the Universe: a conversation over ice cream" [10]. The topics included dark matter, dark energy, and multi-messenger astrophysics. The second event was on the 21st of August, 2019 with the title "Which flavour do you want your neutrino? A conversation over ice cream" [11]. There we had the chance to learn about the highlights of neutrino physics.
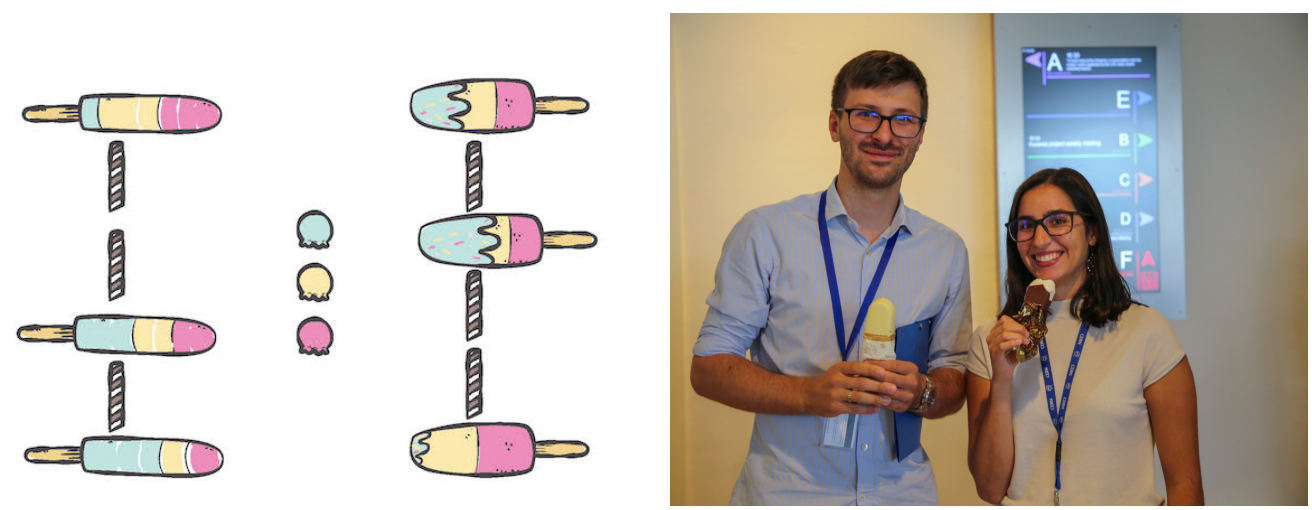

(a)

(b)

Figure 7: (a) The logo of the ice cream event in 2019 about neutrinos and (b) a photo about two participants enjoying their ice cream.

This year it will be on the 16th of June, 2020 and this time we will organize it virtually, over "ice screens". The title thus changes to "A conversation over ice screens: future detectors for particle 
physics" [12]. The invited speakers will describe several future experiments, including NICA, SHiP, ICE-CUBE, and some table-top experiments. In the end of the event we will hear about the future of the field from a representative of the European Commission for Future Accelerators (ECFA).

Regarding the further future plans, we propose a management/leadership training for working group conveners (since they are usually chosen on the physics analysis merit and not on previous leadership experience) by professional trainers. This event was inspired by an ATLAS initiative that was very successful which we believe would be beneficial to extend to the other experiments.

Furthermore, we plan to have an LHC wide mentoring system. ATLAS already has a mentoring system and LHCb's ECGD Office's 2019 survey which concluded that $73 \%$ of the respondents have never had a mentor and they believe that it is useful to have one. The fora thinks that early career scientists could benefit from mentoring from a different experiment. We plan to offer this as an excellence program with limited number of places/duration.

\section{Conclusions}

Despite the differences in the structure and approach in the committees, the goals are common: represent the interests of young scientists and provide a forum to gather and discuss topics relevant to the young LHC community. While at first there were several difficulties to engage the young community, the different events the committees are offering and increased trust in the fora are improving the situation. Several programs are organized either by individual committees or altogether as the young scientist fora. For example, two upcoming events are the next meeting of Soft Skills Workshop series on the 3rd of June, 2020 [6] and the next Ice Cream event (this year over ice screens) on the 17th of June, 2020 [12]. Future plans involve a training for working group conveners and the development of an LHC wide mentoring system.

We have created an e-group where all the LHC young scientist committees can be reached: lhc-early-career-scientists-fora@cern.ch.

If you wish to contact the individual committees, please write to the following e-groups:

- ATLAS Early Career Scientist Board (ECSB): atlas-ecsb@cern.ch

- LHCb Early Career, Gender and Diversity Office (ECGD): 1hcb.ecgd@cern.ch

- CMS Young Scientist Committee (YSC): cms-young-scientist-committee@cern.ch

- ALICE Junior Representatives: alice-junior-reps@ cern.ch

\section{References}

[1] Statistical information about ALICE members https://glance.cern.ch/alice/ membership/members/member_statistics.php

[2] Statistical information about ATLAS members https://atlas.web.cern.ch/Atlas/ GROUPS/PHYSICS/PLOTS/GEN-2019-001/

[3] Statistical information about CMS members https://cms-users.web.cern.ch/ cms-users/cms/Management/Stats/members .html 
[4] Numbers for LHCb come from their membership database, extracted on May 8, 2020

[5] LHC early career scientists fora, "LHC Soft skills workshop: making the best of working from home", https://indico.cern.ch/event/906623/

[6] LHC early career scientists fora, "LHC Soft skills workshop: CV-writing for academia and industry", https://indico.cern.ch/event/914790/

[7] CERN Alumni Relations, "Moving out of academia to ... MedTech", https://indico. cern.ch/event/884493/

[8] LHC early career scientists fora and CERN Alumni, "ALICE, ATLAS, CMS and LHCb Career Networking Event 2019", https://indico.cern.ch/event/843605/

[9] LHC early career scientists fora and CERN Alumni, "ALICE, ATLAS, CMS and LHCb Career Networking Event 2020", https://indico.cern.ch/event/924607/

[10] LHC early career scientists fora, "A fresh look at the Universe: a conversation over ice cream", https://indico.cern.ch/event/737196/

[11] LHC early career scientists fora, "Which flavour do you want your neutrino? A conversation over ice cream", https://indico.cern.ch/event/838486/

[12] LHC early career scientists fora, "A conversation over ice screens: future detectors for particle physics", https://indico.cern.ch/event/914311/ 